\title{
HIGHLIGHT
}

\section{Reprogrammed astrocytes with old 'memories' blossom into region-specific neurons}

\author{
Anuja Ghorpade ${ }^{\bowtie}$ \\ Department of Cell Biology and Anatomy, University of North Texas Health Science Center, 3500 Camp Bowie Blvd, Fort Worth, \\ TX 76107, USA \\ \ Correspondence: Anuja.Ghorpade@unthsc.edu
}

Almost a decade after Dolly the sheep changed the history of biological clock turning, Takahashi and Yamanaka made headlines both in scientific journals, and in magazines such as TIME, by once again proving that the clock could be turned back, even on somatic cells, by 'direct reprogramming.' (Takahashi and Yamanaka, 2006; Park, 2007; Takahashi et al., 2007) During the last decade, stem cells have been at the forefront of science, politics and the public forums due to the conflicting issues surrounding the use of embryonic stem cells in research and regenerative medicine strategies.

The findings of Takahashi and Yamanaka were hailed as the end to the controversy surrounding the use of embryonic stem (ES) cells, as these inducible programmed stem cells were derived by a process called 'direct reprogramming' of somatic cells. In addition, the simplicity of the methods involved made the process accessible to any and all scientists, allow it to be patient-specific and circumvent the issues of immune rejection. Since the original report, over the past few years both mouse and human somatic cells have been successfully transformed into pluripotent stem cells by overexpressing stem cell-specific transcription factors. A combination of four nuclear transcription factors: octamerbinding protein 4 (Oct3/4), SRY-box containing factor 2 (Sox2), Krüppel-like factor 4 (KIf4) and c-Myc, can reprogram differentiated somatic cells to pluripotent embryonic stem celllike state, i.e. inducible programmed stem (iPS) cells. These iPS cells are morphologically and functionally similar to mouse or human embryonic stem cells (Takahashi et al., 2007; Yu et al., 2006; Yu et al., 2007). Both iPS and ES cells can theoretically be propagated indefinitely and have the potential to differentiate into many cell types.

The study by Tian et al. (Tian et al., in this issue) used mouse astrocytes as parental cell to generate iPS cells. A variety of cell types have been reprogrammed into iPS cells including fibroblasts (Takahashi and Yamanaka, 2006;
Takahashi et al., 2007; Yu et al., 2007), neural progenitor cells (Kim et al., 2008; Kim et al., 2009a; Kim et al., 2009b; Kim et al., 2009c), hepatocytes and gastric epithelial cells (Aoi et al., 2008), CD34 + T cells (Ye et al., 2009), B cells (Hanna et al., 2008; Pereira et al., 2008), pancreatic $\beta$ cells (Stadtfeld et al., 2008), melanocytes (Utikal et al., 2009) and keratinocytes (Aasen et al., 2008; Carey et al., 2009). Thus, mouse astrocyte is yet another unique cell type added to the growing list of somatic cell types that can be reprogrammed to pluripotency. However, the addition of astrocytes to the list has significantly broader implications, as these are the most abundant type of neural cells in the human brain and play a critical role in neurodegenerative diseases. Tian et al. also demonstrate that while from the same origin, neural progenitor cells and astrocytes are distinct in their requirements for reprogramming. While neural progenitor cells can be reprogrammed with Oct4 alone or Oct4 and Klf4 in combination, astrocytes require all four of the Yamanaka factors.

The potential that astrocytes may be imparted the abilities to be pluripotent has profound significance in the quest for treatment of neurodegenerative disorders. Indeed, Tian et al. (Tian et al., in this issue) compared neuronal differentiation between mouse astrocyte-derived iPS cells and mouse embryonic fibroblast-derived iPS cells, and found that mouse astrocytes-derived iPS cells possessed greater potential for neuronal differentiation. This is consistent with the notion that the differentiation potential for directly reprogrammed pluripotent cells is influenced by their epigenetic 'memory.' Since reprogramming involves alteration in methylation, some of the epigenetic 'memory' retained is characteristic of their somatic tissue of origin; thus favoring their differentiation along related lineages (Hu et al., 2010; Kim et al., 2010; Polo et al., 2010). During development, gliogenesis precedes neurogenesis with some overlapping time periods, and thus it makes biological sense that glial cells 
harbor heightened potential for differentiating into neuronal cells with direct reprogramming.

It is of greater significance that the iPS cells derived from mouse astrocytes could differentiate into dopaminergic neurons. This makes Tian et al.'s study (Tian et al., in this issue) even more novel and uniquely significant. Clearly, the overall goal of any stem cell research program is to translate the research into therapeutic options thus transitioning from bench to bedside. This is of particular importance in the case of neurodegenerative diseases, as the treatment options for many such diseases are limited and cures remain elusive. An important caveat in the regenerative medicine field is the limited capacity of neural stem cells to generate regionspecific neuronal phenotypes other than the inhibitory olfactory bulb neuronal populations. The adult subventricular zone neural stem cells are primarily destined to efficiently generate the olfactory bulb interneuronal populations, which are non-dopaminergic GABA-ergic interneurons (Lois and Alvarez- Buylla, 1994; Caldwell et al., 2001; Carleton et al., 2003; Jain et al., 2003). Tian et al. successfully demonstrate that mouse astrocyte-derived iPS cells could not only differentiate into the three germ layers in vivo proving their pluripotency, but they also possess high potential for regionspecific dopaminergic neuronal differentiation. Parkinson's disease (PD) is the second most common neurodegenerative disorder, afflicting over six million people worldwide (Schapira, 1999). PD is caused by the loss of dopaminergic neurons in the pars compacta region of the substantia nigra. The findings by Tian et al. (Tian et al., in this issue) provide an important step in the ongoing quest for fundamental changes in neurodegenerative disease therapeutic approaches.

The comparison between mouse astrocytes- and mouse embryonic fibroblast-derived iPS cells showed yet another intricacy in the complexity of this phenomenon. Early passages of the mouse astrocyte-derived iPS cell lines exhibited significantly smaller embryonic bodies compared to those of the mouse embryonic fibroblast-derived iPS cell lines. However, later passages of both cells showed fewer differences. This suggests that not only are the two cells intrinsically distinct in their preferences for differentiation targets, but that the characteristics of the cells are dependent on their development ex vivo. Does this involve a modification of the epigenetic memory along the way? Since time is the age-old impetus for fading memories, are epigenetic memories no exception? If yes, what regulates this process and how will this be influenced in vivo? These issues may have further implications into developing iPS cells into therapeutic avenues.

All unique scientific works not only add to the existing knowledge, further the field significantly by the contribution per se; but they also enhance the future of the field by opening up the next set of questions, just begging to be answered. Tian et al.'s study (Tian et al., in this issue) clearly fits this trend. While it is clear that mouse astrocytic epigenetic memory' may allow astrocytes to evolve into region-specific neurons, what mechanisms are involved in regulating this phenomenon? Is it possible that this memory may fade over time, depending on the influence of other exogenous and/or endogenous factors? The authors articulate very well that specific epigenetic pattern comparisons would be key to first describing their unique features, and then characterizing the mechanisms. In addition, the current study uses retroviral vectors to express the Yanakawa cocktail of transcription factors. Yet another step forward in the development of iPS cells into therapeutic avenues would be to express the reprogramming transcription factors using strategies that will facilitate translational applications. However, the most important question about iPS cells and their therapeutic potential remains their teratogenicity, i.e. these cells form tumors in immunodeficient animals such as SCID mice. While Tian et al. have employed this feature of mouse astrocyte-derived iPS cells to successfully document their pluripotency by development of all three germ layers when implanted in SCID mice, the issues surrounding the teratogenic potential of iPS cells will be an important next question to address prior to therapeutic application. The step that will bring us closest to iPS cell therapy would be: to work out the carefully controlled, one hundred percent differentiation of iPS cells into the desired targets in vivo.

So, are we close yet to using iPS cells as therapeutic agents for neurodegenerative diseases such as Parkinson's disease? The nature of research is that it is incremental, and with every step we get closer. The current study by Tian et al. is a significant step forward, with the clear demonstration that cells, innate to the central nervous system, specifically the astrocytes, hold onto their epigenetic memory and can be directly reprogrammed to become dopaminergic neurons. Thus, this study moves us a critical step closer to the application of iPS cells to neurodegenerative disease therapy, and move them from the bench to the bedside.

While it is a dream for all basic scientists to influence the health of mankind through discoveries and clinical application, yet another exciting component for scientists is the quest for deeper understanding of biology. For glial biologists, this study opens up a novel avenue by utilizing astrocytes, the numerically superior cells of the brain, as a model for direct programming and understanding mechanisms involved in reprogramming of glial cells into neurons. The uncovering of these pathways in glial cells will not only broaden the knowledge of glial biology but provide unique model systems for screening of compounds that may be applied in the future for direct reprogramming of other cells into region-specific neurons that may be better amenable to therapy. Thus, Tian et al. have not only uncovered the unique potential of astrocytes with epigenetic memories that allow for a greater potential for neuronal development, but this study also opens up exciting new questions and areas of cutting-edge research into both stem cell and glial cell biology. 


\section{REFERENCES}

Aasen, T., Raya, A., Barrero, M.J., Garreta, E., Consiglio, A., Gonzalez, F., Vassena, R., Bilić, J., Pekarik, V., Tiscornia, G., et al. (2008). Efficient and rapid generation of induced pluripotent stem cells from human keratinocytes. Nat Biotechnol 26, 1276-1284.

Aoi, T., Yae, K., Nakagawa, M., Ichisaka, T., Okita, K., Takahashi, K., Chiba, T., and Yamanaka, S. (2008). Generation of pluripotent stem cells from adult mouse liver and stomach cells. Science 321 , 699-702.

Caldwell, M.A., He, X., Wilkie, N., Pollack, S., Marshall, G., Wafford, K.A., and Svendsen, C.N. (2001). Growth factors regulate the survival and fate of cells derived from human neurospheres. Nat Biotechnol 19, 475-479.

Carey, B.W., Markoulaki, S., Hanna, J., Saha, K., Gao, Q., Mitalipova, M., and Jaenisch, R. (2009). Reprogramming of murine and human somatic cells using a single polycistronic vector. Proc Natl Acad Sci U S A 106, 157-162.

Carleton, A., Petreanu, L.T., Lansford, R., Alvarez-Buylla, A., and Lledo, P.M. (2003). Becoming a new neuron in the adult olfactory bulb. Nat Neurosci 6, 507-518.

Hanna, J., Markoulaki, S., Schorderet, P., Carey, B.W., Beard, C., Wernig, M., Creyghton, M.P., Steine, E.J., Cassady, J.P., Foreman, R., et al. (2008). Direct reprogramming of terminally differentiated mature B lymphocytes to pluripotency. Cell 133, 250-264.

$\mathrm{Hu}$, Q., Friedrich, A.M., Johnson, L.V., and Clegg, D.O. (2010). Memory in induced pluripotent stem cells: reprogrammed human retinal-pigmented epithelial cells show tendency for spontaneous redifferentiation. Stem Cells 28, 1981-1991.

Jain, M., Armstrong, R.J., Tyers, P., Barker, R.A., and Rosser, A.E. (2003). GABAergic immunoreactivity is predominant in neurons derived from expanded human neural precursor cells in vitro. Exp Neurol 182, 113-123.

Kim, J.B., Greber, B., Araúzo-Bravo, M.J., Meyer, J., Park, K.I., Zaehres, H., and Schöler, H.R. (2009a). Direct reprogramming of human neural stem cells by OCT4. Nature 461, 649-3.

Kim, J.B., Sebastiano, V., Wu, G., Araúzo-Bravo, M.J., Sasse, P., Gentile, L., Ko, K., Ruau, D., Ehrich, M., van den Boom, D., et al. (2009b). Oct4-induced pluripotency in adult neural stem cells. Cell 136, 411-419.

Kim, J.B., Zaehres, H., Araúzo-Bravo, M.J., and Schöler, H.R. (2009c). Generation of induced pluripotent stem cells from neural stem cells. Nat Protoc 4, 1464-1470.

Kim, J.B., Zaehres, H., Wu, G., Gentile, L., Ko, K., Sebastiano, V., Araúzo-Bravo, M.J., Ruau, D., Han, D.W., Zenke, M., et al. (2008). Pluripotent stem cells induced from adult neural stem cells by reprogramming with two factors. Nature 454, 646-650.

Kim, K., Doi, A., Wen, B., Ng, K., Zhao, R., Cahan, P., Kim, J., Aryee, M.J., Ji, H., Ehrlich, L.I., et al. (2010). Epigenetic memory in induced pluripotent stem cells. Nature 467, 285-290.

Lois, C., and Alvarez-Buylla, A. (1994). Long-distance neuronal migration in the adult mammalian brain. Science 264, 1145-1148.

Park, A. (2007). A Breakthrough on Stem Cells. In: TIME (Time Inc.). E-pub. Tuesday, Nov. 20. http://www.time.com/time/health/article/ 0,8599,1685965,00.html

Pereira, C.F., Terranova, R., Ryan, N.K., Santos, J., Morris, K.J., Cui, W., Merkenschlager, M., and Fisher, A.G. (2008). Heterokaryonbased reprogramming of human $B$ lymphocytes for pluripotency requires Oct4 but not Sox2. PLoS Genet 4, e1000170.

Polo, J.M., Liu, S., Figueroa, M.E., Kulalert, W., Eminli, S., Tan, K.Y., Apostolou, E., Stadtfeld, M., Li, Y., Shioda, T., et al. (2010). Cell type of origin influences the molecular and functional properties of mouse induced pluripotent stem cells. Nat Biotechnol 28, 848-855.

Schapira, A.H. (1999). Science, medicine, and the future: Parkinson's disease. BMJ 318, 311-314.

Stadtfeld, M., Brennand, K., and Hochedlinger, K. (2008). Reprogramming of pancreatic beta cells into induced pluripotent stem cells. Curr Biol 18, 890-894.

Takahashi, K., Okita, K., Nakagawa, M., and Yamanaka, S. (2007). Induction of pluripotent stem cells from fibroblast cultures. Nat Protoc 2, 3081-3089.

Takahashi, K., and Yamanaka, S. (2006). Induction of pluripotent stem cells from mouse embryonic and adult fibroblast cultures by defined factors. Cell 126, 663-676.

Tian, C., Wang, Y., Sun, L., Ma, K., and Zheng, J. (2011). Reprogrammed Mouse Astrocytes Retain a "Memory" of Tissue Origin and Possess More Tendencies for Neuronal Differentiation than Reprogrammed MEFs. Protein Cell (in this issue).

Utikal, J., Maherali, N., Kulalert, W., and Hochedlinger, K. (2009). Sox 2 is dispensable for the reprogramming of melanocytes and melanoma cells into induced pluripotent stem cells. J Cell Sci 122, 3502-3510.

Ye, Z., Zhan, H., Mali, P., Dowey, S., Williams, D.M., Jang, Y.Y., Dang, C.V., Spivak, J.L., Moliterno, A.R., and Cheng, L. (2009). Humaninduced pluripotent stem cells from blood cells of healthy donors and patients with acquired blood disorders. Blood 114, 5473-5480.

Yu, J., Vodyanik, M.A., He, P., Slukvin, I.I., and Thomson, J.A. (2006). Human embryonic stem cells reprogram myeloid precursors following cell-cell fusion. Stem Cells 24, 168-176.

Yu, J., Vodyanik, M.A., Smuga-Otto, K., Antosiewicz-Bourget, J., Frane, J.L., Tian, S., Nie, J., Jonsdottir, G.A., Ruotti, V., Stewart, R., et al. (2007). Induced pluripotent stem cell lines derived from human somatic cells. Science 318, 1917-1920. 